\title{
28 Research Square \\ Dietary Carbohydrate Intake and New-onset Diabetes: A Nationwide Cohort Study in China
}

\section{Chun Zhou}

Southern Medical University Nanfang Hospital

\section{Zhuxian Zhang}

Southern Medical University Nanfang Hospital

Mengyi Liu

Southern Medical University Nanfang Hospital

Yuanyuan Zhang

Southern Medical University Nanfang Hospital

Huan Li

Southern Medical University Nanfang Hospital

Panpan He

Southern Medical University Nanfang Hospital

Qinqin Li

Anhui Medical University

Chengzhang Liu

Anhui Medical University

Xianhui Qin ( $\sim$ pharmaqin@126.com )

Southern Medical University Nanfang Hospital https://orcid.org/0000-0001-7812-7982

\section{Original investigation}

Keywords: Carbohydrate intake, High-quality carbohydrate, Low-quality carbohydrate, Low-carbohydrate diet, New-onset diabetes.

Posted Date: June 8th, 2021

DOl: https://doi.org/10.21203/rs.3.rs-551817/v1

License: (a) (i) This work is licensed under a Creative Commons Attribution 4.0 International License. Read Full License 


\section{Abstract}

Background: The association of carbohydrate intake with diabetes risk remains uncertain. We aimed to evaluate the prospective associations of the amount and types of carbohydrate intake with new-onset diabetes.

Methods: A total of 16,260 non-diabetic participants from the China Health and Nutrition Survey (CHNS) were included. Dietary intake was collected by three consecutive 24-h dietary recalls combined with a household food inventory. Participants with self-reported diabetes, or fasting plasma glucose $\geq 7.0$ $\mathrm{mmol} / \mathrm{L}$ or glycated hemoglobin $\geq 6.5 \%$ during the follow-up were defined having new-onset diabetes.

Results: During a median follow-up of 9 years (158,930 person-years), 1,100 participants developed diabetes. Overall, there was a U-shaped association between percent of energy from carbohydrate intake and new-onset diabetes, with minimal risk at $49-56 \%$ of energy from total carbohydrate intake (quartile 2) ( $P$ for nonlinearity $<0.001)$. Moreover, there was an L-shaped association between high-quality carbohydrate intake and new-onset diabetes ( $P$ for nonlinearity $<0.001)$, and a J-shaped association of low-quality carbohydrate intake with new-onset diabetes ( $P$ for nonlinearity $<0.001)$. Furthermore, there was an inverse association between the plant-based low-carbohydrate scores for low-quality carbohydrate and new-onset diabetes. However, a reversed $\mathrm{J}$-shaped association was found between the animal-based low-carbohydrate scores for low-quality carbohydrate and new-onset hypertension ( $P$ for nonlinearity $<0.001)$.

Conclusions: There was a U-shape association between percent of total carbohydrate intake and newonset diabetes, with the lowest risk at $49-56 \%$ carbohydrate intake. Our findings provide some evidence for the intake of high-quality carbohydrate, and the substitution of plant-based products for low-quality carbohydrate for primary prevention of diabetes.

\section{Background}

Diabetes has become a major health concern worldwide due to its high prevalence and related huge burden of disability and mortality. In 2014, global age-standardized diabetes prevalence was $9.0 \%$ in men, and $7.9 \%$ in women ${ }^{1}$. As such, it is of great clinical and public health importance to identify more modifiable related factors for the primary prevention of diabetes.

International Diabetes Federation (IDF) has emphasized that a healthy diet could be the major preventive measure for diabetes ${ }^{2}$. Along with fats and protein, carbohydrate is one of the three basic macronutrients. A low-carbohydrate, high-protein, high-fat diet has been suggested as a strategy for weight-loss ${ }^{3-5}$. However, the results regarding the association of carbohydrate intake with diabetes risk are still considerably mixed, including no significant association in most of the studies in western countries, and positive association in some of the Asian countries ${ }^{6,7}$. The possible explanations may be that the amount and type of carbohydrate intake differ by geographical and socioeconomic factors. 
Western Asian countries usually have a higher percentage of energy from carbohydrate (about 60\%), whereas North American and European countries capture lower carbohydrate (usually $<50 \%{ }^{8}$. Moreover, the dietary patters in Asian countries are substantially different from patterns in Western countries. However, to date, few previous studies have comprehensively examined the amount and type of carbohydrates with incident diabetes. More importantly, few related studies, using the carbohydrate intake data continuously, have been conducted, which may allow for the possibility of non-linear relation of carbohydrate intake and new-onset diabetes.

Considering the above knowledge gaps and the need to provide more evidence for the development of guidelines on amount and type of carbohydrate intake, we aimed to explore the relationships of the amount and type of carbohydrate intake with incident diabetes, and to assess whether replacement carbohydrate with protein and fat affected the risk of diabetes, using data from the China Health and Nutrition Survey (CHNS), a national health and nutrition survey in China.

\section{Materials And Methods}

\section{Study design and participants}

Details on the study design, sampling methods, response rates and some results of the CHNS have been published elsewhere ${ }^{9-13}$. Briefly, CHNS is an ongoing multipurpose longitudinal cohort study initiated in 1989, and has been followed up every 2-4 years. A multistage, random cluster approach was used to draw the sample from 9 provinces (from north to south) and 3 largest autonomous cities in mainland China. The CHNS rounds have been completed in 1989, 1991, 1993, 1997, 2000, 2004, 2006, 2009, 2011 and 2015. In each survey round, demographic, socioeconomic, lifestyle, nutritional and health information were collected.

In this analysis, we conducted a prospective cohort study using seven waves (1997 to 2015) of the CHNS data. In this cohort, members were surveyed in at least two study rounds, and the first survey round is considered as baseline. We excluded participants who were pregnant, <18 years old, or having diabetes at baseline. Participants with missing dietary carbohydrate data or with extreme dietary energy data (male: $>4200$ or <600 kcal/day; female: $>3600$ or $<500$ kcal/day) were further excluded ${ }^{14}$. Finally, 16,260 participants were included in this analysis (eFigure 1).

The institutional review boards of the University of North Carolina at Chapel Hill and the National Institute of Nutrition and Food Safety, and Chinese Center for Disease Control and Prevention, approved the study. Each participant provided their written informed consent.

\section{Dietary nutrient intakes}

Dietary measurements in CHNS are described in detail elsewhere ${ }^{15}$. Briefly, both individual and household level data were collected in each survey round. Dietary information was collected by 3-day dietary recalls through questionnaires, in combination with using a 3-day food-weighed method to assess 
cooking oil and condiment consumption. The three consecutive days were randomly allocated from Monday to Sunday and are almost equally balanced across the seven days of the week for each sampling unit. Nutrient intakes were calculated using the China food composition tables (FCTs). The accuracy of 24-hour dietary recall designed to assess energy and nutrient intake has been validated ${ }^{15}$.

In this study, three-day average intakes of dietary macronutrients and micronutrients in each round were calculated. We evaluated energy-adjusted nutrient intake for each nutrient using sex specific linear regression models ${ }^{16}$. Cumulative average intake values of each nutrient were calculated for each participant, using all results up to the last visit prior to the date of new-onset diabetes, or using all results among those without new-onset diabetes, to reduce within-subject variation and represent long-term dietary intake and minimize within-person variation.

\section{Definition of the low-carbohydrate diet (LCD) scores}

In our present study, macronutrients were divided into high- and low-quality carbohydrate, plant-based protein and fat, as well as animal-based protein and fat. Food sources constituting these subtypes are shown in the eTable $1^{17,18}$.

Low-carbohydrate diet (LCD) scores was defined following an established method ${ }^{19}$. First, the study participants were divided into 11 strata each of fat, protein, and carbohydrate intake, expressed as a percentage of energy (eTable 2). For fat and protein, participants in the highest strata received 10 points for that macronutrient and participants in the lowest strata received 0 points. For carbohydrate, the lowest strata received 10 points and the highest 0 points. The scores of three macronutrients were summed to create the overall LCD scores, which ranged from 0-30 (a higher score reflects less intake of carbohydrate and more intake of fat and protein, while a lower score reflects more intake of carbohydrate and less intake of fat and protein).

A total LCD score for low-quality carbohydrate was built based on percentage of energy intake from lowquality carbohydrate, total protein and total fat. Furthermore, we also built a plant-based LCD score for low-quality carbohydrate, based on percentage of energy intake from low-quality carbohydrate, plant protein and plant fat; and an animal-based LCD score for low-quality carbohydrate based on percent of energy from low-quality carbohydrate, animal protein and animal fat. As such, each participant was given the overall, plant-based and animal-based scores. (eTable 1, eTable 2). In detail, a higher total LCD score reflects less intake of low-quality carbohydrate and more intake of total fat and total protein, a higher plant-based LCD score reflects less intake of low-quality carbohydrate and more intake of plant fat and plant protein, and a higher animal-based LCD score reflects less intake of low-quality carbohydrate and more intake of animal fat and animal protein.

\section{Assessments of blood pressure and covariates}

After the participants had rested for 5 minutes, seated blood pressure was measured by trained research staff using a mercury manometer, following the standard method. Triplicate measurements on the same 
arm were taken in a quiet and bright room. The mean systolic blood pressure (SBP) and diastolic blood pressure (DBP) of the three independent measures were used in analysis.

Demographic and lifestyle information was obtained through questionnaires, including age, sex, smoking status, alcohol consumption, occupation, education level, residence, regions, and concomitant diseases. Body height and weight were measured following a standard procedure with calibrated equipment. Body mass index (BMI) was calculated as weight $(\mathrm{kg})$ by height squared $\left(\mathrm{m}^{2}\right)$. Physical activity was collected by staff-administered questionnaires exploring all occupational, transportation, domestic and leisure activities in adults.

\section{Study outcome}

The participants had been asked to report their diabetes status with a questionnaire-based interview at each follow-up. New-onset diabetes were confirmed if the answer was "yes" to the question "has a doctor ever told you that you suffer from diabetes?". In addition, blood samples were collected and assayed only in 2009. Therefore, in 2009, outcome was also ascertained by an additional criterion: fasting blood glucose $\geq 7.0 \mathrm{mmol} / \mathrm{L}$ or glycated hemoglobin $[\mathrm{HbA} 1 \mathrm{c}] \geq 6.5 \%)^{20,21}$.

When a participant was first identified with new-onset diabetes in a following survey, the middle date between this and the nearest survey before was used to calculate the follow-up time. For those free of diabetes in all following surveys, the last survey date was used to calculate the follow-up time.

\section{Statistical Analysis}

Intake of dietary carbohydrate were expressed as the percentage of total energy using nutrient density method ${ }^{22}$ and then categories into quartile $(<49,49-<56,56-<63, \geq 63$ of energy intake from carbohydrate intake). Population characteristics are presented as mean \pm standard deviations (SDs) for continuous variables, and proportions for categorical variables. Differences in population characteristics by quintile of carbohydrate intake were compared using ANOVA tests, or chi-square tests, accordingly.

We used Cox proportional hazards models to calculate hazard ratios (HRs) and 95\% confidence intervals (Cls) for the risk of new-onset diabetes. Model 1 included adjustments for age, sex, and body mass index (BMI). Model 2 included adjustments for age, sex, regions, BMI, smoking status, systolic blood pressure (SBP), diastolic blood pressure (DBP), education level, urban or rural residence, occupation, physical activity, as well as total energy intake, sodium intake, potassium intake and fiber intake. Furthermore, we used a cubic B-spline with 4 knots $(20 \%, 40 \%, 60 \%, 80 \%$ of carbohydrate intake) to display the potentially non-linear relationship of total, high-quality and low-quality carbohydrate, and total, animal-based and plant-based LCD scores for low-quality carbohydrate with new-onset diabetes in a more intuitive way with adjustments for covariates in Model 2.

Moreover, possible modifications of the association between total, high-quality and low-quality carbohydrate intake and new-onset diabetes were evaluated for the following variables: age ( $<60 \mathrm{vs}$. $\geq 60$ 
years), sex, BMI ( $\left.<24 v s . \geq 24 \mathrm{~kg} / \mathrm{m}^{2}\right)$, waist circumference ( $\left.<80 v s . \geq 80 \mathrm{~cm}\right)$, total protein intake ([median] $<12 v s . \geq 12 \%$ of energy), and total fat intake ([median] $<31 v s$. $\geq 31 \%$ of energy), and interactions between subgroups and carbohydrate intake were examined by likelihood ratio testing.

We consider a two-side $P$ value $<0.05$ as statistically significant in all analysis. All statistical analyses were conducted using $\mathrm{R}$ version 3.6.1.

\section{Results}

\section{Baseline characteristics of study participants}

In the final analysis, a total number of 16,260 participants were included, as showed in eFigure 1 . Mean percent of energy intake as total carbohydrate, high-quality carbohydrate and low-quality carbohydrate was $56.0 \%$ (SD: 10.3 ), $6.6 \%$ (SD: 5.2 ), and $46.1 \%$ (SD: 12.6 ), respectively. Notably, low-quality carbohydrate was the main source of carbohydrate intake, which accounted for $82 \%$ of carbohydrate intake.

Table 1 illustrated baseline characteristics of included participants in line with quartile for percent of energy intake from total carbohydrate intake. Mean age of the 16,260 non-diabetic participants was 43 years (SD: 15.3), and 49\% was males. In participants with higher intake of total carbohydrate, younger age, lower levels of SBP, DBP and BMI were also shown. Moreover, they were more likely to be smoker and farmer, had higher physically active levels, less likely to be unemployed, live urban residence, receive high school education. For diets, they consumed less fat, protein and sodium, but more fiber. 
Table 1

Characteristics of study participants by quartiles of energy from carbohydrate intake (\% of energy) ${ }^{\star}$

\begin{tabular}{|c|c|c|c|c|c|}
\hline & \multicolumn{4}{|c|}{ Quartiles of carbohydrate intake, \% of energy } & \multirow{2}{*}{$\begin{array}{l}P \\
\text { value }\end{array}$} \\
\hline & Q1(<49) & Q2(49<56) & Q3(56<63) & $\mathrm{Q} 4(\geq 63)$ & \\
\hline N & 4065 & 4065 & 4065 & 4065 & \\
\hline Male, No. (\%) & $2253(55.4)$ & $2152(52.9)$ & $2015(49.6)$ & 1899 (46.7) & $\begin{array}{l}<.001 \\
0.00\end{array}$ \\
\hline Age, years & $45.6 \pm 15.6$ & $44.2 \pm 15.5$ & $41.9 \pm 14.8$ & $40.8 \pm 14.9$ & $\begin{array}{l}< \\
0.001\end{array}$ \\
\hline Systolic blood pressure, $\mathrm{mmHg}$ & $\begin{array}{l}121.4 \pm \\
17.4\end{array}$ & $\begin{array}{l}120.0 \pm \\
17.2\end{array}$ & $\begin{array}{l}119.0 \pm \\
17.2\end{array}$ & $\begin{array}{l}118.4 \pm \\
17.2\end{array}$ & < \\
\hline $\begin{array}{l}\text { Diastolic blood pressure, } \\
\mathrm{mmHg}\end{array}$ & $78.3 \pm 10.7$ & $77.7 \pm 10.5$ & $77.2 \pm 10.8$ & $76.9 \pm 10.9$ & $\begin{array}{l}< \\
0.001\end{array}$ \\
\hline Body mass index, $\mathrm{kg} / \mathrm{m}^{2}$ & $23.4 \pm 3.4$ & $23.1 \pm 3.4$ & $22.7 \pm 3.2$ & $22.2 \pm 3.1$ & <. \\
\hline Smoking, No. (\%) & $1168(28.8)$ & $1206(29.7)$ & $1316(32.5)$ & $1401(34.6)$ & $\begin{array}{l}< \\
0.001\end{array}$ \\
\hline Alcohol drinking, No. (\%) & $1487(36.9)$ & $1411(35.1)$ & $1422(35.4)$ & 1419 (35.4) & 0.300 \\
\hline Urban residence, No. (\%) & $2304(56.7)$ & $1940(47.7)$ & $1317(32.4)$ & $580(14.3)$ & < \\
\hline Regions, No. (\%) & & & & & < \\
\hline Central & $2246(55.3)$ & 1799 (44.3) & $1687(41.5)$ & $2087(51.3)$ & \\
\hline North & $806(19.8)$ & $900(22.1)$ & $924(22.7)$ & $854(21)$ & \\
\hline South & $1013(24.9)$ & $1366(33.6)$ & $1454(35.8)$ & $1124(27.7)$ & \\
\hline Physical activity, MET-h/wk & $\begin{array}{l}125.6 \pm \\
115.7\end{array}$ & $\begin{array}{l}135.0 \pm \\
117.6\end{array}$ & $\begin{array}{l}163.3 \pm \\
136.5\end{array}$ & $\begin{array}{l}191.3 \pm \\
141.1\end{array}$ & $<0.001$ \\
\hline Occupation, No. (\%) & & & & & < \\
\hline Farmer & $362(8.9)$ & $734(18.2)$ & $1555(38.7)$ & $2677(66.8)$ & \\
\hline Worker & $521(12.9)$ & $584(14.5)$ & $512(12.7)$ & $278(6.9)$ & \\
\hline Unemployed & 1587 (39.2) & $1375(34.1)$ & $991(24.6)$ & 635 (15.8) & \\
\hline Other & 1577 (39) & 1337 (33.2) & $963(23.9)$ & $420(10.5)$ & \\
\hline
\end{tabular}

*Variables are presented as Mean \pm SDs for continuous variables or $\mathrm{N}(\%)$ for categorical variables. 


\begin{tabular}{|c|c|c|c|c|c|}
\hline & \multicolumn{4}{|c|}{ Quartiles of carbohydrate intake, \% of energy } & \multirow{2}{*}{$\begin{array}{l}P \\
\text { value }\end{array}$} \\
\hline & Q1(<49) & Q2(49<56) & Q3(56<63) & $\mathrm{Q} 4(\geq 63)$ & \\
\hline Education, No. (\%) & & & & & $\begin{array}{l}< \\
0.001\end{array}$ \\
\hline Illiteracy & $554(13.8)$ & $704(17.7)$ & $826(20.7)$ & $1153(29.2)$ & \\
\hline Primary school & $517(12.9)$ & $640(16.1)$ & $864(21.7)$ & $1077(27.3)$ & \\
\hline Middle school & $1152(28.7)$ & $1289(32.4)$ & $1396(35.1)$ & $1256(31.9)$ & \\
\hline High school or above & $1788(44.6)$ & $1348(33.9)$ & $896(22.5)$ & $456(11.6)$ & \\
\hline \multicolumn{6}{|l|}{ Dietary intake } \\
\hline Energy, Kcal/day & $\begin{array}{l}2040.3 \pm \\
549.7\end{array}$ & $\begin{array}{l}2117.2 \pm \\
486.4\end{array}$ & $\begin{array}{l}2172.3 \pm \\
470.0\end{array}$ & $\begin{array}{l}2219.7 \pm \\
513.9\end{array}$ & $\begin{array}{l}< \\
0.001\end{array}$ \\
\hline Total carbohydrate, $\%$ of energy & $42.6 \pm 6.1$ & $53.0 \pm 1.9$ & $59.5 \pm 2.0$ & $68.7 \pm 4.3$ & $<.001$ \\
\hline $\begin{array}{l}\text { High-quality carbohydrate, \% of } \\
\text { energy }\end{array}$ & $5.9 \pm 3.9$ & $6.3 \pm 4.3$ & $6.4 \pm 5.0$ & $7.9 \pm 6.8$ & $\begin{array}{l}< \\
0.001\end{array}$ \\
\hline $\begin{array}{l}\text { Low-quality carbohydrate, \% of } \\
\text { energy }\end{array}$ & $31.8 \pm 8.7$ & $43.0 \pm 6.9$ & $50.4 \pm 6.7$ & $59.1 \pm 8.2$ & $<.001$ \\
\hline Total fat, \% of energy & $43.9 \pm 6.7$ & $34.2 \pm 2.8$ & $28.3 \pm 2.6$ & $19.9 \pm 4.4$ & $<.001$ \\
\hline Total protein, \% of energy & $13.5 \pm 3.1$ & $12.7 \pm 2.2$ & $12.1 \pm 1.9$ & $11.4 \pm 1.5$ & $<.001$ \\
\hline Fiber, g/d & $8.8 \pm 5.1$ & $9.9 \pm 4.9$ & $10.8 \pm 5.6$ & $12.6 \pm 6.0$ & $<.001$ \\
\hline Sodium, g/d & $5.4 \pm 3.4$ & $5.1 \pm 2.4$ & $5.0 \pm 2.6$ & $4.8 \pm 3.0$ & $\begin{array}{l}< \\
0.001\end{array}$ \\
\hline Potassium, g/d & $1.7 \pm 0.6$ & $1.6 \pm 0.5$ & $1.6 \pm 0.6$ & $1.7 \pm 0.6$ & $<.001$ \\
\hline Sodium to potassium ratio & $3.6 \pm 2.5$ & $3.3 \pm 1.8$ & $3.2 \pm 1.8$ & $3.1 \pm 2.0$ & $<.001$ \\
\hline
\end{tabular}

\section{Associations of dietary carbohydrate intake (\% of energy) with new-onset diabetes}

Over a median follow-up of 9 years (inter-quartile range (IQR): 4-15 years, 158, 930 person-years), 1100 (6.8\%) participants developed new-onset diabetes, of which, 640 were those with self-reported new-onset diabetes during follow-up period. 
Overall, the association between percent of energy from total carbohydrate intake and new-onset diabetes followed a U-shape ( $P$ for nonlinearity $<0.001$ ): a percentage of energy in $49-56 \%$ (quartile 2 ) from total carbohydrate intake showed the lowest risk of new-onset diabetes (Fig. 1A, Table 2). 
Table 2

Relative risk of new-onset diabetes according to quartiles of energy from carbohydrate intake

\begin{tabular}{|c|c|c|c|c|}
\hline \multirow[t]{2}{*}{ Carbohydrate intake, \% energy } & \multirow[t]{2}{*}{ No. of case } & \multirow[t]{2}{*}{ Person-years } & Model 1 & Model 2 \\
\hline & & & $\mathrm{HR}(95 \% \mathrm{Cl})$ & $\mathrm{HR}(95 \% \mathrm{Cl})$ \\
\hline
\end{tabular}

\section{Total carbohydrate}

Quartile

\begin{tabular}{lllll} 
Q1 $(<49)$ & 238 & 29333 & $1.24(1.03,1.49)$ & $1.20(0.99,1.45)$ \\
\hline Q2(49-<56) & 260 & 39266 & ref & ref \\
\hline Q3(56-<63) & 286 & 44030 & $1.19(0.99,1.42)$ & $1.26(1.05,1.51)$ \\
\hline Q4 $(\geq 63)$ & 316 & 44030 & $1.44(1.21,1.72)$ & $1.67(1.37,2.05)$
\end{tabular}

Category

\begin{tabular}{lllll} 
Q1(<49) & 238 & 29333 & $1.24(1.03,1.49)$ & $1.21(1.00,1.47)$ \\
\hline Q2(49-<56) & 260 & 39266 & ref & ref \\
\hline Q3-4( $\geq 56)$ & 602 & 87721 & $1.31(1.12,1.52)$ & $1.40(1.19,1.66)$
\end{tabular}

\section{High quality carbohydrate}

Quartile

\begin{tabular}{lllll} 
Q1 $(<3)$ & 332 & 36988 & ref & ref \\
\hline Q2(3-<5) & 257 & 44069 & $0.64(0.54,0.75)$ & $0.59(0.49,0.70)$ \\
\hline Q3(5-<8) & 222 & 41343 & $0.51(0.43,0.61)$ & $0.44(0.36,0.53)$ \\
\hline Q4 $(\geq 8)$ & 289 & 36530 & $0.68(0.57,0.80)$ & $0.54(0.44,0.65)$
\end{tabular}

\section{Low quality carbohydrate}

Quartile

\begin{tabular}{lllll} 
Q1(<38) & 224 & 26777 & $1.21(1.01,1.46)$ & $1.19(0.97,1.45)$ \\
\hline Q2(38-<47) & 263 & 39873 & ref & ref \\
\hline Q3(47-<55) & 295 & 45362 & $1.23(1.03,1.47)$ & $1.39(1.16,1.67)$ \\
\hline Q4( $\geq 55)$ & 318 & 46919 & $1.57(1.32,1.88)$ & $1.95(1.60,2.37)$
\end{tabular}

Model 1: Adjusted for age, sex, BMl;

Model 2: Adjusted for age, sex, BMI, region, smoking, SBP, DBP, education, urban or rural residence, occupation, physical activity, sodium intake, potassium intake, fiber intake, and energy intake.

However, an L-shape association was found for high-quality carbohydrate intake ( $P$ for nonlinearity < 0.001) (Fig. 1B). Comparing with those in the 1st quartile of high-quality carbohydrate intake, the 
adjusted HRs $(95 \% \mathrm{Cls})$ were $0.59(0.49,0.70)$ in 2 nd quartile, $0.44(0.36,0.53)$ in 3rd quartile, and $0.54(0.44,0.65)$ in 4 th quartile, respectively (Table 2$)$.

In contrast, there was a J-shape association between low-quality carbohydrate intake and new-onset diabetes ( $P$ for nonlinearity < 0.001$)$ (Fig. 1C). Comparing with those in the 2nd quartile of low-quality carbohydrate intake, the adjusted HRs $(95 \% \mathrm{Cls})$ was $1.19(0.97,1.45)$ in the 1 st quartile and $1.95(1.60$, 2.37 ) in the 4 th quartile, respectively (Table 2 ).

Similar trends were found for the associations of total (eFigure 2A), high-quality (eFigure 2B), and lowquality carbohydrate (eFigure $2 \mathrm{C}$ ) intake with self-reported new-onset diabetes during follow-up period.

\section{Associations of LCD scores for low-quality carbohydrate with new-onset diabetes}

There was a reversed J-shaped association between animal-based LCD scores for low-quality carbohydrate and new-onset diabetes $(P$ for nonlinearity $<0.001)$ : the adjusted HRs were 1.93 $(95 \% \mathrm{Cl}, 1.38-2.70)$ and $1.39(95 \% \mathrm{Cl}, 1.01-1.92)$ for the 1 st and 10 th decile of the LCD scores, respectively, compared with the 8th decile (Fig. 2B, eTable 3). Similar trends were found for the total LCD scores for low-quality carbohydrate (Fig. 2A, eTable 3).

However, for plant-based LCD scores for low-quality carbohydrate, the adjusted HRs ( $95 \% \mathrm{Cls}$ ) of newonset diabetes was $2.21(1.53-3.20)$ in the 1 st decile and $0.88(0.67-1.17)$ in the 10th decile, compared with those in the 8th decile (Fig. 2C, eTable 3).

\section{Association of dietary fat and protein intake (\% of energy) with new-onset diabetes}

We further examined the association between each macronutrient and new-onset diabetes in multivariate nutrient density models. Overall, there were U-shaped associations of the percentage energy from animal(eFigure $3 \mathrm{~A}$ ) or plant-based (eFigure 3B) fat, animal- (eFigure 3C) or plant-based (eFigure 3D) protein intake with new-onset diabetes (All $P$ for nonlinearity $<0.001$ ).

\section{Stratified Analyses}

In the stratified analyses, age, sex, BMI, waist circumference, the percentage energy from total protein and total fat did not significantly modify the association between the percentage energy from total, highquality and low-quality carbohydrate intake with the risk of new-onset diabetes (All P-interactions $>0.05$ ) (eTable 4).

\section{Discussion}

Findings from a large sample, nation-wide, prospective study in China showed a U-shaped association between percent of energy from total carbohydrate intake and new-onset diabetes, with the minimal risk observed at $49-56 \%$ carbohydrate intake (quartile 2 ). The increased risks of new-onset diabetes were 
mainly found in those with lower intake of high-quality carbohydrate or higher intake of low-quality carbohydrate. Furthermore, there was an L-shape association between the plant-based LCD scores for low-quality carbohydrate and new-onset diabetes. However, there was a reversed J-shaped association between animal-based LCD scores for low-quality carbohydrate and new-onset diabetes.

A previous meta-analysis of eight studies in western countries showed that the pooled estimate of RR was 0.97 (95\% $\mathrm{Cl} 0.90-1.06)$ per $50 \mathrm{~g}$ per day of total dietary carbohydrate intake $(P=0.5)$. However, there was a substantial heterogeneity between the cohort studies ${ }^{6}$. After that, controversial findings had still been reported, with positive associations in obese Japanese men, middle-aged Korean adults, or Chinese women ${ }^{23-25}$, while no associations in Korea or USA population ${ }^{26-28}$. Of note, among Chinese women, the corresponding relative risks (RR) for risk of type 2 diabetes were $0.96,0.87,1.09,1.28$ for participants in quintile 2 , quintile 3 , quintile 4, quintile 5 , respectively, compared those in quintile 1 of carbohydrate intake. That is to say that this study also showed a U-shaped trend between carbohydrate intake and diabetes risk. Overall, the above studies suggested that the association between carbohydrate intake and diabetes risk remains inconclusive. The discrepant findings may be partly due to the difference of the amount and type of carbohydrate intake in different population. Moreover, previous studies, which usually assumed linearity between carbohydrate intake and diabetes, seemed to have overlooked some valuable information.

Based on data of a large-scale sample, multi-center, prospective, nationwide cohort in China, our study provided a chance to examine the continuous association of the amount and type of carbohydrate intake (\% of energy) with new-onset diabetes in a population with a wide range of carbohydrate intake. We provided some new insights in this field. First, there was a U-shaped association of the percentage of energy consumed from total carbohydrate with new-onset diabetes: with the lowest risk observed at 49$56 \%$ (quartile 2) carbohydrate intake. Consistently, previous studies also reported the U-shape associations of percent of energy from carbohydrate intake with mortality ${ }^{14}$ and new-onset hypertension 29 , with lowest incidence risk at $50-60 \%$ and $50-55 \%$ of carbohydrate intake, respectively. Second, the increased new-onset diabetes risk was mainly observed in those with lower intake of high-quality carbohydrate or higher intake of low-quality carbohydrate. Accordingly, a previous study had reported the beneficial effect of high-quality carbohydrate and the detrimental effect of low-quality carbohydrate on the risk of cardiovascular disease and mortality ${ }^{30}$. Ley SH et al also recommended the dietary patterns rich in high-quality carbohydrate and lower in low-quality carbohydrate foods for diabetes prevention and management ${ }^{31}$.

These findings might be in reasonable explication. Extreme low dietary carbohydrate is not recommended because of its essential role in maintaining normal physiological function, including primary fuel use by the brain and central nervous system, sources of water-soluble vitamins and minerals as well as fiber and so on ${ }^{32}$. Moreover, high-quality carbohydrate, including fruits, and non-starch vegetables, etc., is abundant in antioxidants, and phytochemicals. These ingredients can regulate glucose by accelerating hepatic insulin response and decreasing hepatic glucose output in healthy participants ${ }^{33,34}$. However, 
low-quality carbohydrate, including refined grains, starchy vegetables, and sugars, was absorbed mainly as glucose that may possibly easily increase plasma glucose and insulin response ${ }^{35}$.

Furthermore, we found that there was an L-shape association between the plant-based LCD scores for low-quality carbohydrate and new-onset diabetes. Consistently, previous studies had reported inverse associations of LCD scores that favored plant-derived protein and fat intake with the risk of mortality 14,36 , hypertension ${ }^{29}$ and coronary heart diseases ${ }^{19,37}$. Moreover, although a positive association of LCD scores favoring animal-derived protein and fat sources with mortality ${ }^{14}$ had been reported, our study showed that there seemed to be a reversed J-shaped association of animal-based LCD scores for lowquality carbohydrate with new-onset diabetes. On the one hand, these results suggested that due to the high intake of low-quality carbohydrate in this population, the possible unfavorable effect associated with moderate animal fats and proteins intake may be still lower than the detrimental effect of high intake of low-quality carbohydrate intake on the new-onset diabetes. On the other hand, our results really indicated that substitution of plant-based products for low-quality carbohydrate may be a more appropriate strategy for prevention of diabetes.

Some limitations are needed considering. First, due to the observational design, although broad covariates have been included in the adjustments, residual confounding from unmeasured or unrecorded risk factors cannot be excluded. Second, fasting glucose and HbA1c were only available in round 2009, the incident rate of type 2 diabetes might be underestimated. Third, dietary measurements in CHNS were derived from self-reported dietary 24-hour recalls, which may be affected by recall bias. Nevertheless, it's one of the most common methods for dietary intake data, and has been used by some precious important cohorts ${ }^{8,14,22}$. More importantly, the accuracy of 24-hour dietary recall for the evaluation of energy and nutrient intake has been validated by lots of previous studies ${ }^{15,38,39}$. In addition, CHNS was conducted in China, the generalization of our results to other ethnics and population still needs more studies. Further confirmation of our findings in future studies is necessary.

In conclusion, our study indicated that there was a U-shaped association between percentage of dietary carbohydrate intake and new-onset diabetes in general Chinese adults, with minimal risk observed at 49$56 \%$ (quartile 2) of carbohydrate intake. The increased risks were mainly observed in participants with lower intake of the high-quality carbohydrate or higher intake of low-quality carbohydrate. Moreover, there was an L-shaped association between plant-based LCD scores for low-quality carbohydrate and newonset diabetes. If further confirmed, our findings support the intake of high-quality carbohydrate, and the substitution of plant-based products for low-quality carbohydrate for prevention of diabetes.

\section{Abbreviations}

CHNS: China Health and Nutrition Survey; IDF: International Diabetes Federation; FCT: food composition table; LCD: low-carbohydrate diet; SBP: systolic blood pressure; DBP: diastolic blood pressure; BMI: body mass index; HbA1c: glycated hemoglobin; SD:standard deviation; Cl: confidence interval; IQR: interquartile range.

Page $13 / 18$ 


\section{Declarations}

\section{Ethics approval and consent to participate:}

The institutional review boards of the University of North Carolina at Chapel Hill and the National Institute of Nutrition and Food Safety, and Chinese Center for Disease Control and Prevention, approved the study. Each participant provided their written informed consent.

\section{Consent for publication:}

Not applicable.

\section{Availability of data and materials:}

The datasets analysed during the current study are available in the CHNS official website.

\section{Competing interests:}

The authors declare that they have no competing interests.

\section{Funding:}

The study was supported by the National Natural Science Foundation of China [81973133, 81730019], Outstanding Youths Development Scheme of Nanfang Hospital, Southern Medical University (2017J009; X Qin).

\section{Authors' Contributions:}

Chun Zhou, Xianhui Qin, Yuanyuan Zhang, Huan Li, Panpan He, and Qinqin Li designed the research; Chun Zhou, Zhuxian Zhang, Mengyi Liu, and Xianhui Qin conducted the research; Chun Zhou, Chengzhang Liu and Mengyi Liu performed the data management and statistical analyses; Chun Zhou and Xianhui Qin wrote the manuscript; All authors revised and approved the final manuscript.

\section{Acknowledgements:}

This research uses data from China Health and Nutrition Survey (CHNS). We thank the National Institute for Nutrition and Health, China Center for Disease Control and Prevention, Carolina Population Center (P2C HD050924, T32 HD007168), the University of North Carolina at Chapel Hill, the NIH (R01-HD30880, DK056350, R24 HD050924, and R01-HD38700) and the NIH Fogarty International Center (D43 TW009077, D43 TW007709) for financial support for the CHNS data collection and analysis files from 1989 to 2015 and future surveys, and the China-Japan Friendship Hospital, Ministry of Health for support for CHNS 2009, Chinese National Human Genome Center at Shanghai since 2009, and Beijing Municipal Center for Disease Prevention and Control since 2011. 


\section{References}

1. NCD Risk Factor Collaboration (NCD-RisC). Worldwide trends in diabetes since 1980: a pooled analysis of 751 population-based studies with 4.4 million participants. Lancet. 2016;387(10027):1513-1530.

2. https://www.idf.org/aboutdiabetes/type-2-diabetes.html

3. Bazzano LA, Hu T, Reynolds K, et al. Effects of low-carbohydrate and low-fat diets: a randomized trial. Ann Intern Med. 2014; 161:309-318.

4. Chawla S, Tessarolo Silva F, Amaral Medeiros S, Mekary RA, Radenkovic D. The Effect of Low-Fat and Low-Carbohydrate Diets on Weight Loss and Lipid Levels: A Systematic Review and MetaAnalysis. Nutrients. 2020; 12(12): 3774.

5. Boden G, Sargrad K, Homko C, Mozzoli M, Stein TP. Effect of a low-carbohydrate diet on appetite, blood glucose levels, and insulin resistance in obese patients with type 2 diabetes. Ann Intern Med. 2005;142(6):403-11.

6. Greenwood DC, Threapleton DE, Evans CE, et al. Glycemic index, glycemic load, carbohydrates, and type 2 diabetes: systematic review and dose-response meta-analysis of prospective studies. Diabetes Care. 2013;36(12):4166-71.

7. Hardy DS, Garvin JT, Xu H. Carbohydrate quality, glycemic index, glycemic load and cardiometabolic risks in the US, Europe and Asia: A dose-response meta-analysis. Nutr Metab Cardiovasc Dis. 2020;30(6):853-871.

8. Dehghan M, Mente A, Zhang X, et al. Associations of fats and carbohydrate intake with cardiovascular disease and mortality in 18 countries from five continents (PURE): a prospective cohort study. Lancet .2017; 390:2050-2062.

9. Popkin BM, Du S, Zhai F, Zhang B. Cohort Profile: The China Health and Nutrition Survey--monitoring and understanding socio-economic and health change in China, 1989-2011. Int J Epidemiol. 2010;39(6):1435-40.

10. Zhang B, Zhai FY, Du SF, Popkin BM. The China Health and Nutrition Survey, 1989-2011. Obes Rev. 2014;15 Suppl 1:2-7

11. He J, Fang A, Yu S, Shen X, Li K. Dietary Nonheme, Heme, and Total Iron Intake and the Risk of Diabetes in Adults: Results From the China Health and Nutrition Survey. Diabetes Care. 2020;43(4):776-784.

12. Liu M, Zhou C, Zhang Z, et al. Inverse Association Between Riboflavin Intake and New-Onset Hypertension: A Nationwide Cohort Study in China. Hypertension. 2020;76(6):1709-1716.

13. Zhang Z, Liu M, Zhou C, et al. Evaluation of Dietary Niacin and New-Onset Hypertension Among Chinese Adults. JAMA Netw Open. 2021;4(1): e2031669.

14. Seidelmann SB, Claggett $B$, Cheng $S$, et al. Dietary carbohydrate intake and mortality: a prospective cohort study and meta-analysis. The Lancet Public Health. 2018; 3: e419-e428. 
15. Zhai F, Guo X, Popkin B, et al. Evaluation of the 24-hour individual recall method in China. Food Nutr Bull. 1996; 17 (2): 1-5.

16. Willett W, Stampfer MJ. Total energy intake: implications for epidemiologic analyses. Am J Epidemiol. 1986;124(1):17-27.

17. Shan Z, Rehm CD, Rogers G, Ruan M, Wang DD, Hu FB, Mozaffarian D, Zhang FF, Bhupathiraju SN. Trends in dietary carbohydrate, protein, and fat intake and diet quality among US Adults, 1999-2016. JAMA. 2019;322:1178-

18. S. Department of Agriculture (USDA). USDA Choose MyPlate.gov Vegetable Group Food Gallery. Accessed June 8, 2020. https://www.choos[1]emyplate.gov/vegetable-group-food-gallery (8 June 2020).

19. Halton TL, Willett WC, Liu S, et al. Low-carbohydrate-diet score and the risk of coronary heart disease in women. N Engl J Med. 2006; 355:1991-2002

20. Jia W, Weng J, Zhu D, et al. Standards of medical care for type 2 diabetes in China 2019. Diabetes Metab Res Rev. 2019;35(6): e3158.

21. American Diabetes Association. 2. Classification and Diagnosis of Diabetes: Standards of Medical Care in Diabetes-2021. Diabetes Care. 2021 Jan;44(Suppl 1):S15-S33.

22. Hu FB, Stampfer MJ, Rimm E, et al. Dietary fat and coronary heart disease: a comparison of approaches for adjusting for total energy intake and modeling repeated dietary measurements. Am J Epidemiol 1999; 149:531-540.

23. AlEssa HB, Bhupathiraju SN, Malik VS, et al. Carbohydrate quality and quantity and risk of type 2 diabetes in US women. Am J Clin Nutr. 2015;102(6):1543-53.

24. Sakurai M, Nakamura K, Miura K, et al. Dietary carbohydrate intake, presence of obesity and the incident risk of type 2 diabetes in Japanese men. J Diabetes Investig. 2016;7(3): 343-51.

25. Villegas R, Liu S, Gao YT, et al. Prospective study of dietary carbohydrates, glycemic index, glycemic load, and incidence of type 2 diabetes mellitus in middle-aged Chinese women. Arch Intern Med. 2007;167(21):2310-6.

26. Lee KW, Lyu J, Park JK, Jo C, Kim SS. Dietary carbohydrate quality and quantity in relation to the incidence of type 2 diabetes: A prospective cohort study of middle-aged and older Korean adults. Nutrition. 2019; 57:245-251.

27. Ha K, Joung H, Song Y. Inadequate fat or carbohydrate intake was associated with an increased incidence of type 2 diabetes mellitus in Korean adults: A 12-year community-based prospective cohort study. Diabetes Res Clin Pract. 2019; 148: 254-261.

28. Kim SY, Woo HW, Lee YH, et al. Association of dietary glycaemic index, glycaemic load, and total carbohydrates with incidence of type- 2 diabetes in adults aged $\geq 40$ years: The Multi-Rural Communities Cohort (MRCohort). Diabetes Res Clin Pract. 2020; 160:108007.

29. Li Q, Liu C, Zhang S, et al. Dietary Carbohydrate Intake and New-Onset Hypertension: A Nationwide Cohort Study in China. Hypertension. 2021. doi: 10.1161/HYPERTENSIONAHA.120.16751. [Online ahead of print.] 
30. Ho FK, Gray SR, Welsh P, et al. Associations of fat and carbohydrate intake with cardiovascular disease and mortality: prospective cohort study of UK Biobank participants. BMJ. 2020;368:m688.

31. Ley SH, Hamdy O, Mohan V, Hu FB. Prevention and management of type 2 diabetes: dietary components and nutritional strategies. Lancet. 2014;383(9933):1999-2007.

32. Institute of Medicine of the National Academies: Dietary Reference Intakes for Energy, Carbohydrate, Fiber, Fat, Fatty Acids, Cholesterol, Protein, and Amino Acids (Macronutrients). Washington, DC, National Academy Press, 2002.

33. Thorburn A, Muir J, Proietto J. Carbohydrate fermentation decreases hepatic glucose output in healthy subjects. Metabolism. 1993; 42:780-785.

34. Weickert MO, Mohlig M, Koebnick C, et al. Impact of cereal fibre on glucoseregulating factors. Diabetologia. 2005; 48:2343-2353.

35. Wolever TM, Miller JB. Sugars and blood glucose control. Am J Clin Nutr. 1995;62(1 Suppl):212S$221 \mathrm{~S}$.

36. Campmans-Kuijpers MJ, Sluijs I, Nöthlings U, et al. Isocaloric substitution of carbohydrates with protein: the association with weight change and mortality among patients with type 2 diabetes. Cardiovasc Diabetol. 2015;14:39.

37. Salie R, Huisamen B, Lochner A. High carbohydrate and high fat diets protect the heart against ischaemia/reperfusion injury. Cardiovasc Diabetol. 2014;13:109.

38. Xue H, Yang M, Liu Y, et al. Relative validity of a 2-day 24-hour dietary recall compared with a 2-day weighed dietary record among adults in South China. Nutr Diet. 2017; 74:298-307.

39. Yao M, McCrory MA, Ma G, et al. Relative influence of diet and physical activity on body composition in urban Chinese adults. Am J Clin Nutr. 2003; 77:1409-1416.

\section{Figures}
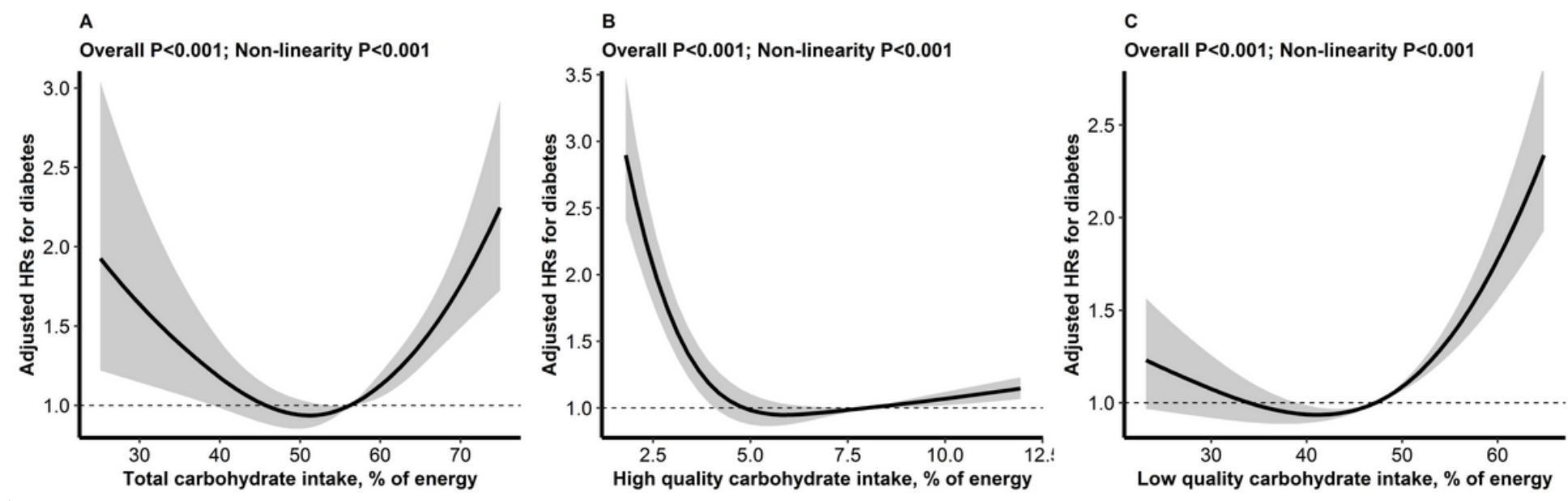

Figure 1 
Associations between percent of energy from carbohydrate intake and new-onset diabetes (A: total carbohydrate, B: high-quality carbohydrate, C: low-quality carbohydrate) * * Adjusted for age, sex, BMI, region, smoking, SBP, DBP, education, urban or rural residence, occupation, physical activity, sodium intake, potassium intake, fiber intake, and energy intake.
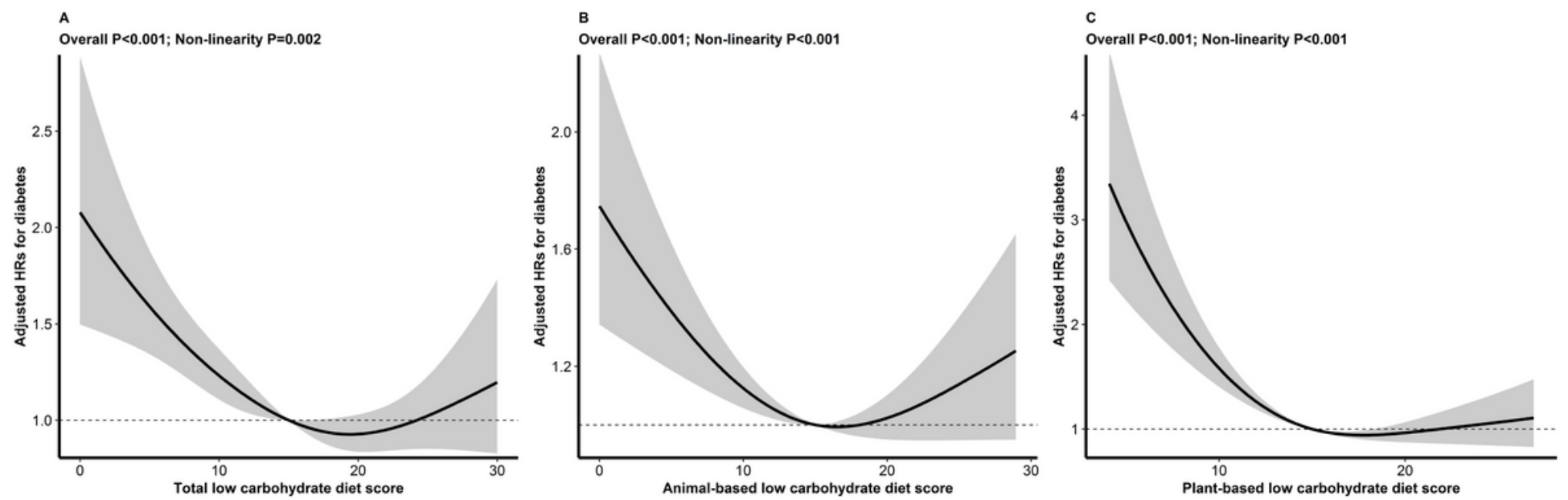

Figure 2

Associations between low-carbohydrate diet (LCD) scores for low-quality carbohydrate and new-onset diabetes (A: total LCD scores, B: animal-based LCD scores, C: plant-based LCD scores) * *Adjusted for age, sex, BMI, region, smoking, SBP, DBP, education, urban or rural residence, occupation, physical activity, sodium intake, potassium intake, fiber intake, and energy intake.

\section{Supplementary Files}

This is a list of supplementary files associated with this preprint. Click to download.

- chodmSupplementaryAppendix0521.doc 